\title{
Blood vessels in neurological development and disease: more than silent spectators
}
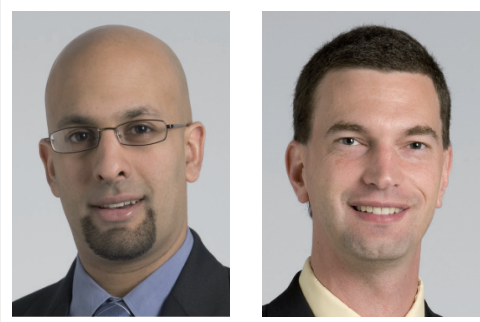

"The extent to which new vessel formation directly impacts stem cell proliferation and survival is not fully understood..."

Justin D Lathia' \& Jeremy N Rich ${ }^{\dagger 1}$

'Department of Stem Cell Biology \& Regenerative Medicine, Cleveland Clinic, 9500 Euclid Avenue, Mailstop NE30, Cleveland, OH 44195, USA

†Author for correspondence: = Tel.: +1 2166360790 = Fax: +1 2166365454 w richj@ccf.org

Neurologists have long faced limitations in their abilities to treat their patients owing to the inability to stimulate regrowth or repair in brain tissue. Although work continues on therapies directed towards healing neurons and supportive brain cells, there may be benefit in focusing on a new target: the vasculature. Blood vessels have been heavily studied in cerebrovascular diseases and brain tumors owing to their roles in the pathophysiology of these diseases, but blood vessels have complex roles in development and repair, suggesting that vascular modulation may have therapeutic benefits in a broad spectrum of neurologic conditions. This is notable, as a range of pharmacologic regulators of blood vessels are already approved for a number of indications and many more are in development. A focused approach to understanding the brain vasculature represents a collaborative opportunity for clinical and fundamental science colleagues to work towards appreciating vascular changes as they relate to brain disorders and the development of brain-regeneration strategies.

From the early 1900 s, one of the central dogmas of neuroscience, put forth by Santiago Ramon y Cajal, was that regeneration did not occur within the brain. In the 1960s, autoradiographical labeling demonstrated that mitotic cells were present in the adult mammalian brain [1], and work examining the seasonal changes in songbird behavior in the early 1980s demonstrated active neurogenesis [2]. These findings have spurred neurogenesis research and have opened up the possibility of neural stem cell therapies for regenerative medicine. Work over the last decade has highlighted diverse neural stem/ progenitor cell populations present within the embryonic and adult brain [3]. Several of these cell populations, namely those residing in neural stem cell niches within the adult hippocampus and adjacent to the lateral ventricles, depend on the existing vasculature [4]. Communication with the vasculature via mitogenic signals, cellto-cell contact and interaction between cells and the extracellular matrix on blood vessels regulate neural stem cell behavior [5]. Similarities and distinctions between blood vessels adjacent to neural stem cells compared with other blood vessels throughout the brain, and the influence of neural stem cells on blood vessels are less well defined. Elucidating these relationships as they relate to disease processes represents a good starting point in the development of repair strategies that incorporate blood vessels.

"Communication with the vasculature via mitogenic signals, cell-to-cell contact and interaction between cells and the extracellular matrix on blood vessels regulate neural stem cell behavior..."

Neural stem cells were first described in basal homeostasis but neural stem cells also respond to brain injury and pathology. While the change in the vascular component of the niches has been studied less, the mobilization of neural stem cells in response to physiological changes appears to rely on interactions with blood vessels. Work in experimental stroke models has shown that mobilized neural stem cells utilize communication with the existing vessels to migrate from their niche to areas of injury [6]. In addition, these mobilized stem cells interact with both existing and newly formed vessels. The extent to which new vessel formation directly impacts stem cell proliferation and survival is not fully understood, but it is likely that mechanisms present for vascular growth and

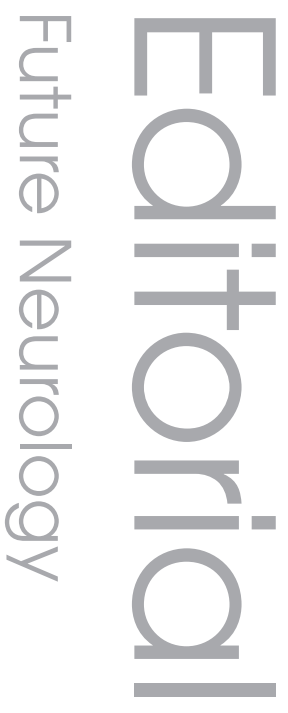

\section{Keywords \\ - blood vessel $=$ brain repair - stem cells

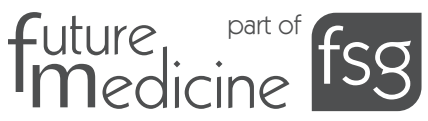


remodeling impact neural stem cell behavior. Neural stem cells and endothelial cells may share some molecular characteristics, and this may be of benefit in the development of regeneration strategies [7]. However, in therapeutic paradigms where the vasculature is targeted, there may be unexpected toxicities, such as cognitive defects reported for the use of the anti-VEGF antibody bevacizumab (Avastin ${ }^{\circledR}$; Genentech/Roche, CA, USA) for the treatment of brain tumor patients. Another major point of consideration for the vascular relationship of neural stem cells is their use in transplantation for regenerative medicine applications. To date, evidence suggests that transplanted neural stem cells used for lineagetracing analysis or in regeneration paradigms do not preferentially localize to vascular niches or blood vessels in non-niche areas. How these stem cells integrate into a new environment without preferentially localizing to a vascular niche is currently a limitation in regenerative medicine, and may help explain the limited success of CNS transplantation and provide additional areas of investigation to help increase transplant survival and efficiency.

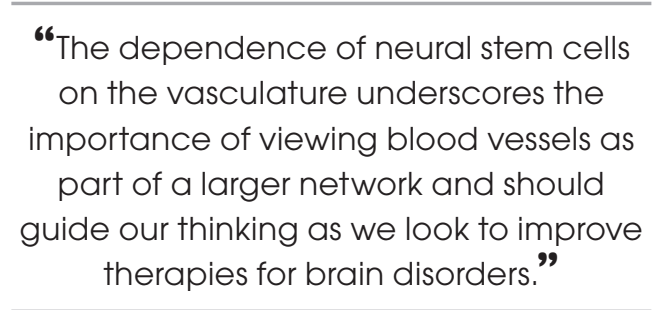

Brain tumors have been another major area that has benefited from a focus on the stem cellblood vessel interaction. In several malignant brain tumors (including glioblastoma multiforme $[\mathrm{GBM}]$ ), it has been demonstrated that stem-like cancer cells reside in proximity to blood vessels [8]. These stem-like cancer cells are of particular interest as they have been shown to be therapeutically resistant and capable of generating a secondary tumor upon transplantation into an animal model [9]. With regards to the vascular relationships described from normal neural stem cells, stem-like cancer cells also communicate with blood vessels in a bidirectional manner [10]. While many additional studies will be required to establish conserved and unique interactions between the vasculature in neural stem cells and stem-like cancer cells, this relationship has been evaluated as a potential therapeutic target. Recently, bevacizumab has been approved by the US FDA for use in recurrent GBMs. While showing promising results in preclinical models and early-phase clinical trials $[8,11,12]$, bevacizumab has not been consistently effective and there are reports that recurrent tumors show a more invasive phenotype [13], underscoring both the critical and complex role of blood vessels in GBMs. There are a variety of reasons why bevacizumab may not be universally effective (e.g., heterogeneity of the tumor, intrinsic resistance and vascular compensation) [14], but the development of an invasive phenotype suggests that there are other aspects of the stem-like cancer cell-blood vessel relationship that may not rely solely on VEGF signaling. Future studies evaluating how bevacizumab-treated tumors develop an invasive phenotype are likely to uncover additional sites of therapeutic intervention that may be used in combination with bevacizumab for more effective GBM therapies.

Looking forward, the bidirectional relationship with the vasculature is central to the regulation of neural stem cells and should be an important consideration in the design of regeneration paradigms. Additional studies defining the contribution of the vasculature to maintenance of neural stem cells and feedback from the stem cells of the vasculature will better guide the development of interventions for brain traumas, degeneration and malignancies. Comparisons with other organs where a similar relationship is present between the vasculature and stem cells may identify additional pathways for consideration and allow the determination of potential systemic consequences of targeting this relationship in the brain. The dependence of neural stem cells on the vasculature underscores the importance of viewing blood vessels as part of a larger network and should guide our thinking as we look to improve therapies for brain disorders.

\footnotetext{
Financial \& competing interests disclosure Work in Jeremy N Rich's laboratory is supported by the the James S McDonnell Foundation, the Goldhirsh Foundation and NIH grants (NS047409, NS054276, CA112958 and CA116659). Jeremy N Rich is a Damon Runyon-Lilly Clinical Investigator supported by the Damon Runyon Cancer Research Foundation. Justin D Lathia is supported by an American Brain Tumor Association Basic Research Fellowship (sponsored by the Joelle Syverson Fund) and a National Service Research Award (NCI F32 CA142159). The authors have no other relevant affliations or financial involvement with any organization or entity with a financial interest in or financial conflict with the subject matter or materials discussed in the manuscript apart from those disclosed.

No writing assistance was utilized in the production of this manuscript.
} 


\section{Bibliography}

1. Altman J, Chorover SL: Autoradiographic investigation of the distribution and utilization of intraventricularly injected adenine- $3 \mathrm{~h}$, uracil- $3 \mathrm{~h}$ and thymidine- $3 \mathrm{~h}$ in the brains of cats. J. Physiol. 169, 770-779 (1963).

2. Goldman SA, Nottebohm F: Neuronal production, migration, and differentiation in a vocal control nucleus of the adult female canary brain. Proc. Natl Acad. Sci. USA 80(8), 2390-2394 (1983).

3. Kriegstein A, Alvarez-Buylla A: The glial nature of embryonic and adult neural stem cells. Annu. Rev. Neurosci. 32, 149-184 (2009).

4. Goldberg JS, Hirschi KK: Diverse roles of the vasculature within the neural stem cell niche. Regen. Med.4(6), 879-897 (2009).
5. Lathia JD, Rao MS, Mattson MP, Ffrench-Constant C: The microenvironment of the embryonic neural stem cell: lessons from adult niches? Dev. Dyn. 236(12), 3267-3282 (2007).

6. Kojima T, Hirota Y, Ema M et al.: Subventricular zone-derived neural progenitor cells migrate along a blood vessel scaffold toward the post-stroke striatum. Stem Cells 28(3), 545-554 (2010).

7. Androutsellis-Theotokis A, Rueger MA, Park DM et al.: Targeting neural precursors in the adult brain rescues injured dopamine neurons. Proc. Natl Acad. Sci. USA 106(32), 13570-13575 (2009).

8. Calabrese C, Poppleton H, Kocak M et al.: A perivascular niche for brain tumor stem cells. Cancer Cell 11, 69-82 (2007).

9. Reya T, Morrison SJ, Clarke MF, Weissman IL: Stem cells, cancer, and cancer stem cells. Nature 414, 105-111 (2001).
10. Gilbertson RJ, Rich JN: Making a tumour's bed: glioblastoma stem cells and the vascular niche. Nature Rev. 7(10), 733-736 (2007).

11. Bao S, Wu Q, Sathornsumetee $S$ et al.: Stem cell-like glioma cells promote tumor angiogenesis through vascular endothelial growth factor. Cancer Res. 66(16), 7843-7848 (2006).

12. Vredenburgh JJ, Desjardins A, Herndon JE 2nd et al:: Bevacizumab plus irinotecan in recurrent glioblastoma multiforme. J. Clin. Oncol. 25(30), 4722-4729 (2007).

13. Norden AD, Young GS, Setayesh K et al.: Bevacizumab for recurrent malignant gliomas: efficacy, toxicity, and patterns of recurrence. Neurology 70(10), 779-787 (2008).

14. Bergers G, Hanahan D: Modes of resistance to anti-angiogenic therapy. Nature Rev. 8(8), 592-603 (2008). 